\title{
Sistema informático para el análisis de los indicadores turísticos en el Hotel Habana Libre
}

\author{
DOI: https://doi.org/10.33262/ap.v2i2.29
}

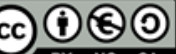

Computer system for the analysis of tourist indicators at the Hotel Habana Libre

Yasser Vázquez Alfonso., ${ }^{1}$ Osvaldo J. Sánchez Arenas., ${ }^{2}$ Leandro Ricardo Pérez. ${ }^{3}$ \& Luis Efraín Velasteguí López. ${ }^{4}$
\end{abstract}

\begin{abstract}
.
The analysis of the relevant information in the tourism and other sectors is a priority concern of many managers both nationally and internationally, since in recent years there has been a great interest in looking for mechanisms to analyze the performance of the different units Organizational Group Hotel Gran Caribe and Hotel Habana Libre is taken as a place for the development of research. Due to this it is proposed to elaborate the project to solve this existing problem where one of the objectives that was raised was to develop a computer application that allows the analysis of the relevant information, starting from this is that the SIAIO software arises. It was implemented in the Java Programming Language on the NetBeans 7.0 IDE and the PostgreSQL 9.1 Database Management System, using the RUP software development methodology with its UML modeling language for the preparation of several modules such as: management of all the information, the calculation that includes the determination of the indices for performance at a general level, by organizational units, the
\end{abstract}

\footnotetext{
${ }^{1}$ Facultad de Turismo, Universidad de la Habana, Cuba. yalfos1@gmail.com

${ }^{2}$ Facultad de Turismo, Universidad de la Habana, Cuba. yalfos1@gmail.com

${ }^{3}$ Facultad de Turismo, Universidad de la Habana, Cuba. yalfos1@gmail.com

${ }^{4}$ Consorcio Ciencia Digital, Ecuador. luisefrainvelaztegui@cienciadigital.org
} 
obtaining of the rules of association with the decision tree and the graphical one. This research is of great importance so that the different managers can make decisions and improve the performance of the different organizational units within the facility.

Keywords: relevant analysis, organizational units, Habana Libre, SIAIO.

\section{Resumen.}

El análisis de la información relevante en el sector del turismo y otros es una preocupación prioritaria de muchos directivos tanto a nivel nacional como internacional, ya que en los últimos años ha surgido un gran interés en buscar mecanismos que permitan analizar el desempeño de las diferentes unidades organizativas del Grupo Hotelero Gran Caribe y se toma como lugar para el desarrollo de la investigación el Hotel Habana Libre. Debido a esto se propone elaborar el proyecto para resolver esta problemática existente donde uno de los objetivos que se plantearon fue desarrollar una aplicación informática que permita el análisis de la información relevante, partiendo de esto es que surge el software SIAIO. El mismo fue implementado en el Lenguaje de Programación Java sobre el IDE NetBeans 7.0 y el Sistema Gestor de Base de Datos PostgreSQL 9.1, utilizando la metodología de desarrollo de software RUP con su lenguaje de modelado UML para la confección de varios módulos como son: el de gestión de toda la información, el de cálculo que incluye la determinación de los índices por desempeño a nivel general, por unidades organizativas, la obtención de las reglas de asociación con el árbol de decisión y el de gráfica. Esta investigación resulta de gran importancia para que los diferentes directivos puedan tomar decisiones y mejorar el desempeño de las distintas unidades organizativas dentro de la instalación.

Palabras claves: análisis relevantes, unidades organizativas, Habana Libre, SIAIO.

\section{Introducción.}

En la actualidad el servicio alojamiento es una de las actividades comerciales más importantes del planeta. Para la Organización Mundial del Turismo (OMT, 2016) en los albores del nuevo milenio, el turismo se consolida como la principal actividad económica de muchos países y el sector de más rápido crecimiento en términos de ingresos de divisas $\mathrm{y}$ creación de empleo, generador de exportaciones del mundo y un factor importante en la balanza de pagos de muchos países. Según el secretario general de la OMT (2016a), Francesco Frangialli, el turismo mantiene la economía mundial "en movimiento", siendo la primera partida del comercio internacional, generando el mayor volumen de ingresos por exportaciones y dando empleo a millones de personas, producto de su carácter multisectorial, y pese a las continuas turbulencias mundiales que amenazan su expansión y que se ha mantenido tenazmente, por ello muchos países se interesan seriamente en su desarrollo. 
Incluso, el turismo, en las últimas décadas ha configurado la geografía, demografía, economía y aspectos socio-culturales en muchas regiones del mundo.

Las ciencias de la computación y la informática han brindado soporte al desarrollo de la tecnología y han aportado soluciones novedosas al tratamiento de la información. De manera general, el uso de estas en las organizaciones empresariales y en la toma de decisiones ha permitido alcanzar nuevos horizontes en la competitividad internacional en muchas áreas y especialmente en el turismo.

En este sentido los indicadores de gestión cobran una gran importancia para la mejora en el desempeño institucional; sin embargo, su diseño e implementación implican en la mayoría de los casos un desafío, dada la complejidad de las entidades que quieren aplicarlos.

Según (Ballester, 2012) la medición de la gestión global de una entidad requiere del desarrollo de un conjunto armónico y sistemático de indicadores que abarquen, con un adecuado conocimiento de sus posibles interrelaciones, las dimensiones de economía, eficacia, eficiencia y calidad del servicio. Además, la medición de los mismos está condicionada por la capacidad de la institución de generar la información necesaria y de elaborar los indicadores, con niveles adecuados de calidad, certeza y confiabilidad.

En cualquier organización, los sistemas de indicadores, dan seguimiento a la estrategia trazada por la empresa. (Ferrer y Gamboa, 2004). Los índices deben proporcionar la información necesaria para la toma de decisiones en los distintos niveles gerenciales, siendo un criterio clave en la medición de los componentes del turismo. Los sistemas de indicadores van a representar la base para una toma de decisiones más acertada y acorde con la realidad, a partir de la cual es posible determinar las acciones a seguir, tomando en cuenta las variaciones experimentadas por los indicadores.

A nivel internacional se reportan numerosas instituciones las cuales se enfocan en realizar aplicaciones informáticas para la toma de decisiones entre estas se destacan: Programa de las Naciones Unidas (PNUD), InduSoft (Industria del software de Argentina), Red para la Sociedad de la Información (RSI-Perú), Dimensión Informática Software Libre (España) entre otros (Pérez; 2020).

En cuba a partir de 1997 se realizan las primeras instalaciones de un software de origen portugués NewHotel. Con un diseño novedoso fue el primer sistema instalado que usaba a plenitud las bondades y facilidades del entorno Microsoft Windows y una poderosa base de datos en Oracle. Este llegó a emplearse en alrededor de 30 hoteles y unos 15 restaurantes. Sin duda NewHotel fue el sistema que más mostró avances en su época, por el amplio diapasón de sus soluciones y la novedad que incorporó a las mismas. 
Posteriormente surgió Zun, un desarrollo más moderno, con una concepción totalmente nacional, además de pasar a un ambiente moderno y con MySQL como gestor de base de datos. Zun mejoró significativamente su apariencia y funcionalidad respecto a NewHotel, llegando a ser el Sistema de Gestión Hotelera (SGH) de mayor presencia en las instalaciones hoteleras de Cuba. Los SGH son sistemas estratégicos para las cadenas cubanas y el MINTUR, pues la información que registran constituye la fuente de gran parte de la estadística general sobre los arribos de turistas, sus gustos, los aspectos de calidad, estudios de segmentación, satisfacción, repitencia, reacciones a las rebajas, comportamiento de las Agencias del MINTUR, Cuentas por Cobrar internacionales; aspectos de los que se ocupa el MINTUR.

En la actualidad la demanda de servicios turísticos se encuentra creciendo y de ahí que las organizaciones turísticas cubanas tengan un papel fundamental en la gestión de la información turística ofrecida al usuario final con el objetivo de aumentar la calidad del sector turístico de nuestro país. La información que se gestiona en estas entidades es de suma importancia para la organización, sin embargo, observamos que no se realiza un correcto manejo de la misma, ya no se apoyan en las ventajas que ofrecen las nuevas tecnologías.

La necesidad de digitalizar la información en el Hotel Habana Libre, en el ámbito nacional, ha traído consigo en diferentes áreas la creación de software para resolver los problemas en la toma de decisiones, por lo que la informatización es fundamental para administrar operaciones, mejorar la eficiencia y obtener ventajas comparativas. La técnica de administración tradicional implica un freno para el desarrollo más avanzado de las unidades organizativas y su economía. Es por eso que el objetivo de esta investigación es desarrollar un sistema informático para el análisis de los indicadores turísticos en el Hotel Habana Libre.

\section{Materiales y métodos.}

Hotel Tryp Habana Libre es uno de los hoteles más grandes de Cuba. Localizado en Vedado, La Habana, cuenta con 572 habitaciones distribuidas por 25 pisos: 532 Doble Estándar, 37 Yúnior Suit Estándar, 1 Suit Presidencial y 2 Sénior Suit Estándar.

El hotel fue construido como Havana Hilton con un presupuesto de 24 millones de dólares bajo los auspicios personales del presidente Fulgencio Batista, como una inversión en el plan de pensiones de la Unión de Trabajadores de Cuba, siendo operado por la cadena estadounidense Hilton Hotels. El hotel fue diseñado por el conocido arquitecto de Los Ángeles, Welton Becket, quien anteriormente había diseñado el Beverly Hilton para la cadena estadounidense, en colaboración del arquitecto Nicolás "Lin" Arroyo2 y Gabriela Menéndez. Arroyo fue el ministro de Obras Públicas de Batista.3

Inaugurado el 22 de marzo de 1958, contó con la presencia de Conrad Hilton, después de cuatro días de festejos.4 En su momento, el Havana Hilton fue el hotel más alto y grande de 
toda Latinoamérica. Contaba con un restaurante Trader Vic's, casino, sala de comedor, sala de billar y un bar en la azotea del hotel.

Este hotel utiliza el sistema de información ejecutiva que es el más adecuado a su forma de operar y emplea como medio para el análisis de la información el sistema de nóminas que tiene como objetivo:

1. Elaborar un modelo conceptual de la gestión de la información brindada por el departamento.

2. Diseñar la base de datos necesaria

3. Diseñar e implementar una aplicación con una interface amigable para este fin.

4. Lograr que el sistema emita todos los reportes necesarios sobre las nóminas de pago

5. Garantizar la seguridad dentro del sistema.

Los métodos utilizados en la investigación son:

\section{Métodos Teóricos.}

Análisis histórico lógico. Lo histórico está relacionado con el estudio de la trayectoria real de los fenómenos y acontecimientos en el de cursar de una etapa o período. Lo lógico se ocupa de investigar las leyes generales del funcionamiento y desarrollo del fenómeno, estudia su esencia. Lo lógico y lo histórico se complementan y vinculan mutuamente. Para poder descubrir las leyes fundamentales de los fenómenos, el método lógico debe basarse en los datos que proporciona el método histórico, de manera que no constituya un simple razonamiento especulativo. De igual modo lo histórico no debe limitarse sólo a la simple descripción de los hechos, sino también debe descubrir la lógica objetiva del desarrollo histórico del objeto de investigación. Este proceso fue utilizado principalmente para toda la bibliografía consultada para poder adquirir los conocimientos necesarios para la realización de esta investigación.

Analítico-Sintético: Este método consiste en la desmembración de un todo, descomponiéndolo en sus partes o elementos para observar las causas, la naturaleza y los efectos y después relacionar cada reacción mediante la elaboración de una síntesis general del fenómeno estudiado. Este proceso se llevó a cabo para poder ver el origen de cada documento e información utilizada dentro de la investigación para así poder conocer las causas de porque fue planteado de esa manera.

Modelación: El modelo científico es un instrumento de la investigación de carácter material o teórico, creado para reproducir el objeto que se está estudiando. Constituye una reproducción simplificada de la realidad que cumple una función heurística que permite descubrir nuevas relaciones y cualidades del objeto de estudio. Un modelo 
científico es la configuración ideal que representa de manera simplificada una teoría. Es un instrumento de trabajo que supone una aproximación intuitiva a la realidad y que tiene por función básica la de ayudar a comprender las teorías y las leyes. La aplicación del método de la modelación está íntimamente relacionada con la necesidad de encontrar un reflejo mediatizado de la realidad objetiva. De hecho el modelo constituye un eslabón intermedio entre el sujeto (investigador) y el objeto de investigación. La modelación es justamente el método mediante el cual se crea abstracciones con vistas a explicar la realidad.

Sistematización: Proceso permanente y acumulativo de creación de conocimientos a partir de las experiencias de intervención en una realidad social. Registrar, de manera ordenada, una experiencia que deseamos compartir con los demás, combinando el quehacer con su sustento teórico, y con énfasis en la identificación de los aprendizajes alcanzados en dicha experiencia. Se empleó este este método con el fin de agrupar todas las experiencias que se tuvo durante la investigación practica que se realizó en la entidad y además de los conocimientos de los entrevistados y del propio investigador.

Análisis Documental: El análisis documental es una forma de investigación técnica, un conjunto de operaciones intelectuales, que buscan describir y representar los documentos de forma unificada sistemática para facilitar su recuperación. Comprende el procesamiento analítico- sintético que, a su vez, incluye la descripción bibliográfica y general de la fuente, la clasificación, indización, anotación, extracción, traducción y la confección de reseñas. Este método se ve presente en el análisis de todos los documentos que se ven presentes en dicha investigación.

Enfoque a sistema: Con el objetivo de lograr la interacción entre los diferentes elementos que conforman el sistema informático elaborado. Este método se ve reflejado en el aspecto programación y en la relación con todos los sistemas que lo componen.

\section{Métodos Nivel Empírico.}

La entrevista: Es la comunicación interpersonal establecida entre el investigador y el sujeto de estudio a fin de obtener respuestas verbales alas interrogantes planteadas sobre el problema propuesto. La entrevista es una técnica de recolección de datos que involucra el cuestionamiento oral de los entrevistados ya sea individualmente o en grupo. Las respuestas a las preguntas durante la entrevista pueden ser registradas por escrito o grabadas en una cinta. Este método se realizó de forma no estandarizada, es decir, a través de una guía con solo lo que se quería conocer. Este fue realizado en la propia instalación.

Consulta a Especialistas: Se aplicó para conocer los diferentes elementos que conforman los indicadores de desempeño en el servicio de alojamiento. Aquí se referencia a dos especialistas de la instalación que son Jefe de Calidad y Subdirector de 
RRHH para así poder obtener la información acerca de los indicadores que se manejan en la entidad.

Consulta de documentos: Fue empleado en la revisión de la literatura especializada y los documentos rectores de la política del MINTUR con respecto al desempeño del servicio de alojamiento. Y para extraer la información necesaria relacionada con el objeto de investigación.

\section{Métodos Estadísticos-Matemáticos.}

Método Estadístico: El fundamento de este método lo constituye la aplicación y el desarrollo de las ideas de la teoría de las probabilidades como una de las disciplinas matemáticas más importantes. Sirve a tres propósitos específicos: Obtener información necesaria, organizar, resumir y presentar en forma adecuada el material numérico, analizar e interpretar los resultados obtenidos.

El método estadístico desempeña entonces una importante función al suministrar un conjunto de métodos y procedimientos sumamente útiles para la investigación, no importa cuál sea la investigación que se realice no la rama del conocimiento humano que se aplique. Este método se ve reflejado en la parte de la programación directamente ya que fue sacado y empleado como parte del software para el logro de los resultados.

Otros procesos que se pretenden automatizar consisten en estructurar el conjunto de indicadores que permiten realizar el análisis del desempeño de las unidades organizativas en cuestión; la clasificación de índices que consiste en aplicar algoritmos de clasificación a la estructura creada donde las fórmulas planteadas por (Sepúlveda, 2008) son usadas en esta investigación para su implementación y sus expresiones son:

$$
x_{i}-\min (x)
$$

a)

$f=\overline{\operatorname{má} x(x)-\min (x)}$ si se aspira a un valor alto del indicador donde:

$f$ : es el valor correspondiente al logro relativo (o índice) en un período determinado.

Xi: representa valor del indicador para la empresa.

mín. X: representa el valor mínimo observado.

máx. X: representa el valor máximo observado. 


\section{$\operatorname{máx}(x)-x_{i}$ \\ b) $\quad f=\overline{\operatorname{máx}(x)-\min (x)}$, si se aspira a un valor bajo del indicador.}

\section{¿Cómo se establecen las ponderaciones para cada indicador?}

Se basa en:

a) La importancia que se le atribuye a cada una de los componentes.

b) La capacidad discriminativa de cada indicador.

Con la condición de que la suma de las ponderaciones sea igual a 1 .

Se parte según López (2004), de que cada uno de los indicadores tiene igual peso en la conformación del índice. Luego, las ponderaciones vienen dadas solamente por las diferencias en la capacidad discriminativa del índice.

Sean $\mathrm{w} 1, \mathrm{w} 2, \ldots, \mathrm{w} 7$ las ponderaciones dadas a cada uno de los siete indicadores integrantes del índice, tal que $\mathrm{w} 1+\mathrm{w} 2+\ldots+\mathrm{w} 7=1$. Sea $\mathrm{k}$ una constante de proporcionalidad asociada a las ponderaciones. Luego, $\mathrm{w} 1=\mathrm{kV} 1, \mathrm{w} 2=\mathrm{kV} 2, \ldots, \mathrm{w} 7=\mathrm{kV} 7$, donde V1, V2,..., V7 pueden ser los coeficientes de variación o la varianza de los logros relativos de cada uno de los indicadores.

Por tanto, $\mathrm{kV} 1+\mathrm{kV} 2+\ldots+\mathrm{kV7}=1$ de lo que resulta que, $\mathrm{k}=1 /(\mathrm{V} 1+\mathrm{V} 2+\ldots+\mathrm{V} 7)$ y el Índice de desempeño $=\mathrm{kv} 1 \mathrm{f} 1+\mathrm{kv} 2 \mathrm{f} 2+\ldots+\mathrm{kvnfn}$

\section{Clasificación del criterio de medida.}

Para el logro de la clasificación del desempeño de las diferentes unidades organizativas se toma la clasificación planteada por (Sandoval et. al., 2007) que clasifica el índice de desempeño en:

- $\quad$ Si Índice $>0,8$ se considera muy bueno

- $\quad$ Si 0,5 < Índice $<0,799$ se considera bueno

- Si Índice $<0,5$ se considera malo

A esta clasificación hay que hacerle un algoritmo. 

abril-junio, 2020

Otros de los procesos que es objeto de automatización es la construcción de gráficos estadísticos como gráfico de barra, líneas, pastel, áreas y curvas de segunda y tercera dimensión.

A continuación, se detallan y refinan los requerimientos funcionales y no funcionales del sistema. También se describirá el estilo arquitectónico que tendrá el sistema, mostrando una agrupación de las funcionalidades en paquetes a alto nivel. Luego se elaborará el diagrama de caso de uso del sistema que representa la interacción de los actores con las funcionalidades del mismo y la descripción de los casos de uso que se automatizarán.

Definición de los requisitos funcionales:

RF-1 Autenticar.

RF-2 Gestionar Usuario.

RF-2.1. Insertar usuario.

RF-2.2. Modificar usuario.

RF-2.3. Eliminar usuario.

RF-2.4. Buscar usuario.

RF-2.5. Listar usuario.

RF-3 Gestionar Unidad Organizativa.

RF-3.1. Insertar unidad.

RF-3.2. Modificar unidad.

RF-3.3. Eliminar unidad.

RF-3.4. Buscar unidad.

RF-3.5. Listar unidad.

RF-4 Gestionar Tipo de Observación.

RF-4.1. Insertar tipo de observación.

RF-4.2. Modificar tipo de observación.

RF-4.3. Eliminar tipo de observación. 
RF-4.4. Buscar tipo de observación.

RF-4.5. Listar tipo de unidades.

RF-5 Gestionar Observación.

RF-5.1. Insertar observación.

RF-5.2. Modificar observación.

RF-5.3. Eliminar observación.

RF-5.4. Buscar observación.

RF-5.5. Listar observación.

RF-6 Gestionar Componentes.

RF-6.1. Insertar componentes.

RF-6.2. Modificar componentes.

RF-6.3. Eliminar componentes.

RF-6.4. Buscar componentes

RF-6.5. Listar componentes.

RF-7 Gestionar Variables de Componentes.

RF-7.1. Insertar variables de componentes.

RF-7.2. Modificar variables de componentes.

RF-7.3. Eliminar variables de componentes.

RF-7.4. Buscar variables de componentes

RF-7.5. Listar variables de componentes.

RF-8 Gestionar Valores.

RF-8.1. Insertar valores.

RF-8.2. Modificar valores. 
RF-8.3. Eliminar valores.

RF-8.4. Buscar valores.

RF-8.5. Listar valores.

\section{Definición de los requisitos no funcionales.}

Los requerimientos no funcionales son propiedades o cualidades que el producto debe cumplir. Debe pensarse en estas propiedades como las características que hacen al producto atractivo, usable, rápido o confiable.

\section{Apariencia o interfaz externa.}

La interfaz de usuario debe ser lo más amigable posible, dado que la mayoría del personal encargado de trabajar con el programa, no está vinculado por lo regular con lo que a informática y computación se refiere. Debe tener colores agradables a la vista, pero a la vez serios para que el enfoque del usuario no se desvíe de la tarea a realizar.

\section{Usabilidad.}

El sistema debe ser de utilización más bien simple, sin dejar de obtener el usuario un buen resultado en la búsqueda de una respuesta que le dé solución al problema en que se encuentra. Se prevé que sea de gran utilización ya que no existe ninguna aplicación como esta.

\section{Rendimiento.}

El sistema debe ser capaz de mantener una buena agilidad en el procesamiento de los datos para así garantizar aceptación y un mayor uso de la aplicación por parte de los usuarios.

\section{Soporte.}

Tener en cuenta que una vez terminado el software es necesario seguir perfeccionamiento el trabajo.

\section{Seguridad.}

Se contará con un servidor encargado de que la información esté protegida contra acceso no autorizado, al tener registrados los usuarios encargados de trabajar con la aplicación y los roles asignados a los mismos. De igual manera cada usuario en dependencia del rol que juegue tendrá distintos permisos de acceso, lo que evitará un mal manejo de la información guardada, así como cambios no deseados en la misma. 


\section{Confiabilidad.}

La información que el sistema maneja está protegida, debido a que solo se le permite la manipulación de esta a personal autorizado.

\section{Portabilidad.}

La utilización de Java como lenguaje de programación y el uso de las herramientas libres y multi plataforma garantice que el sistema sea usado bajo cualquier ambiente.

\section{Software y Hardware.}

El sistema presenta buenas prestaciones en un ordenador P4 con las siguientes características que son consideradas como mínimas: Micro Intel Pentium o compatible a $1.7 \mathrm{GHz}, 512 \mathrm{Mb}$ de RAM, 40 GB de disco duro y Monitor con resolución 800 X 600 pixeles.Debe contar con el paquete instalado JDK 1.6 (Java Development Kit, esta versión al menos) en la Pc cliente y PostgreSQL 9.1 en el servidor.

\section{Resultados y discusión.}

El software SIAIO 1.0 (figura 1), representa un sistema informático que cuenta con una base de datos para almacenar toda la información necesaria en un proceso de análisis de información relevante, contiene algoritmos matemáticos que calculan el índice de desempeño, la clasificación por ese índice de desempeño, obtiene reglas de asociación (Apriori) y clasificación (Nnge), genera árboles de decisión. Nos brinda un gráfico en segunda dimensión que muestra el desempeño de las diferentes unidades organizativas y además nos da la posibilidad de gestionar toda la información que sea entrada por los operadores.

Figura 1: Interfaz principal del software SIAIO1.0

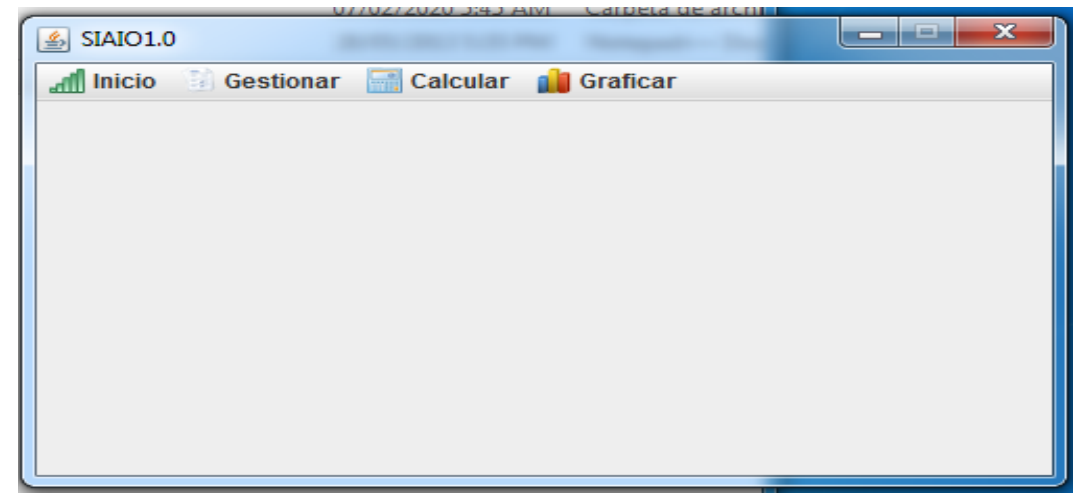

Fuente: Elaboración propia. 
Esta aplicación primeramente muestra una interfaz que le permitirá al usuario ingresar a ella mediante su nombre y su contraseña (figura 2). Mediante esta interfaz de autenticación cada usuario pasará a desempeñar sus actividades según los roles establecidos previamente, lo que le permitirá que acceda solo a los módulos habilitados para cada usuario.

Figura 2: Interfaz de autenticación.

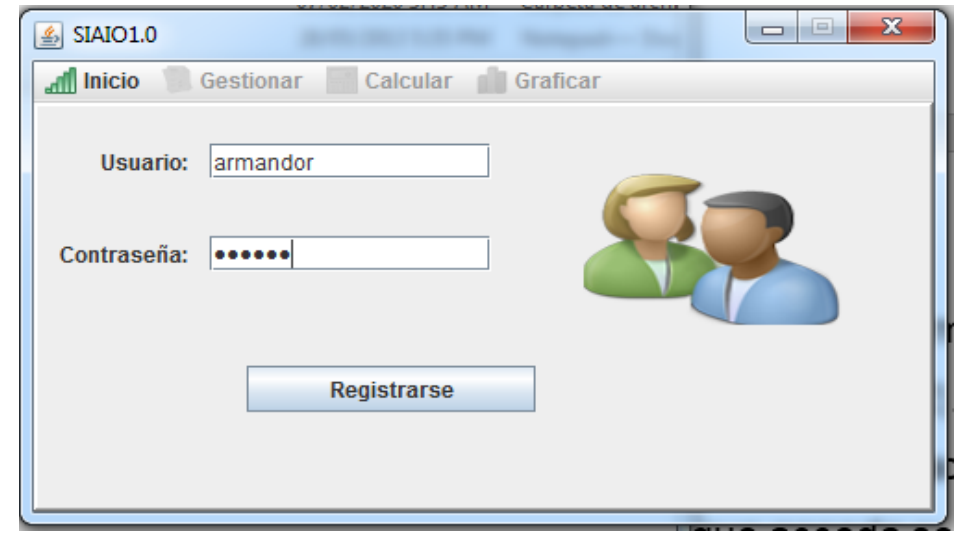

Fuente: Elaboración propia.

Este software (figura 3), nos brinda la posibilidad de poder obtener estos resultados, teniendo en cuenta su unidad organizativa y tipo de observación para mayor facilidad y mejor resultado en la toma de decisiones.

Figura 3: Interfaz de variables por componentes.

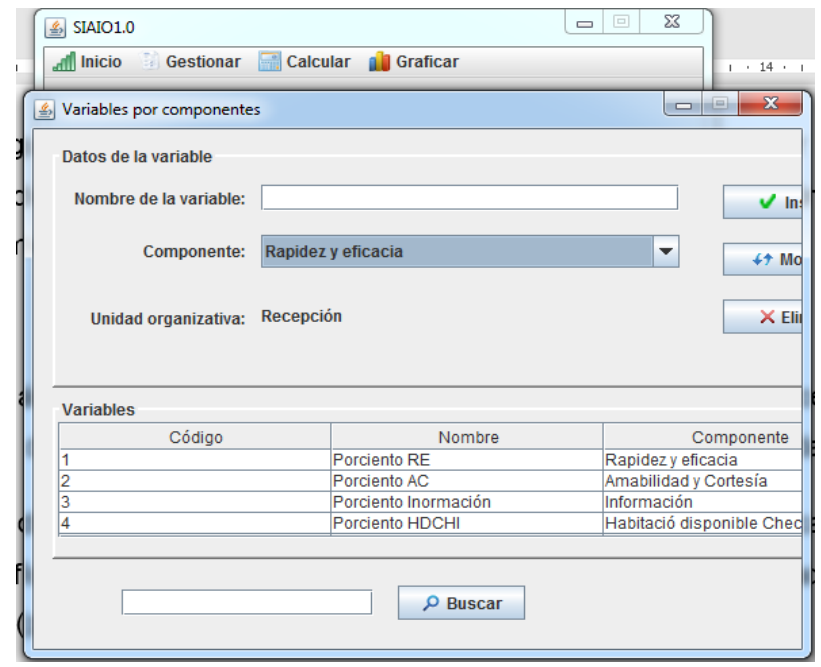

Fuente: Elaboración propia.

El operador además de acceder al módulo de gráfica y cálculo tendrá acceso al módulo de gestión donde podrá gestionar toda la información de la base de datos (ver figura 4). 
Figura 4: Interfaz del módulo gestionar.

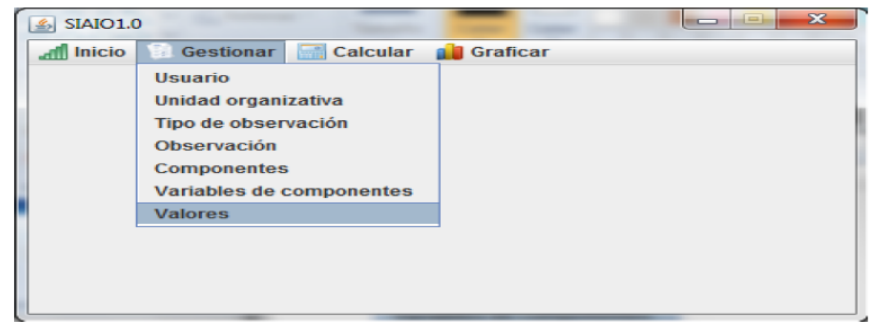

Fuente: Elaboración propia.

La Figura 5 muestra los resultados del cálculo de los índices por unidad organizativa, donde se observa que a medida que van pasando los años el desempeño de esos componentes van mejorando.

Figura 5: Interfaz del cálculo del índice por unidad organizativa.

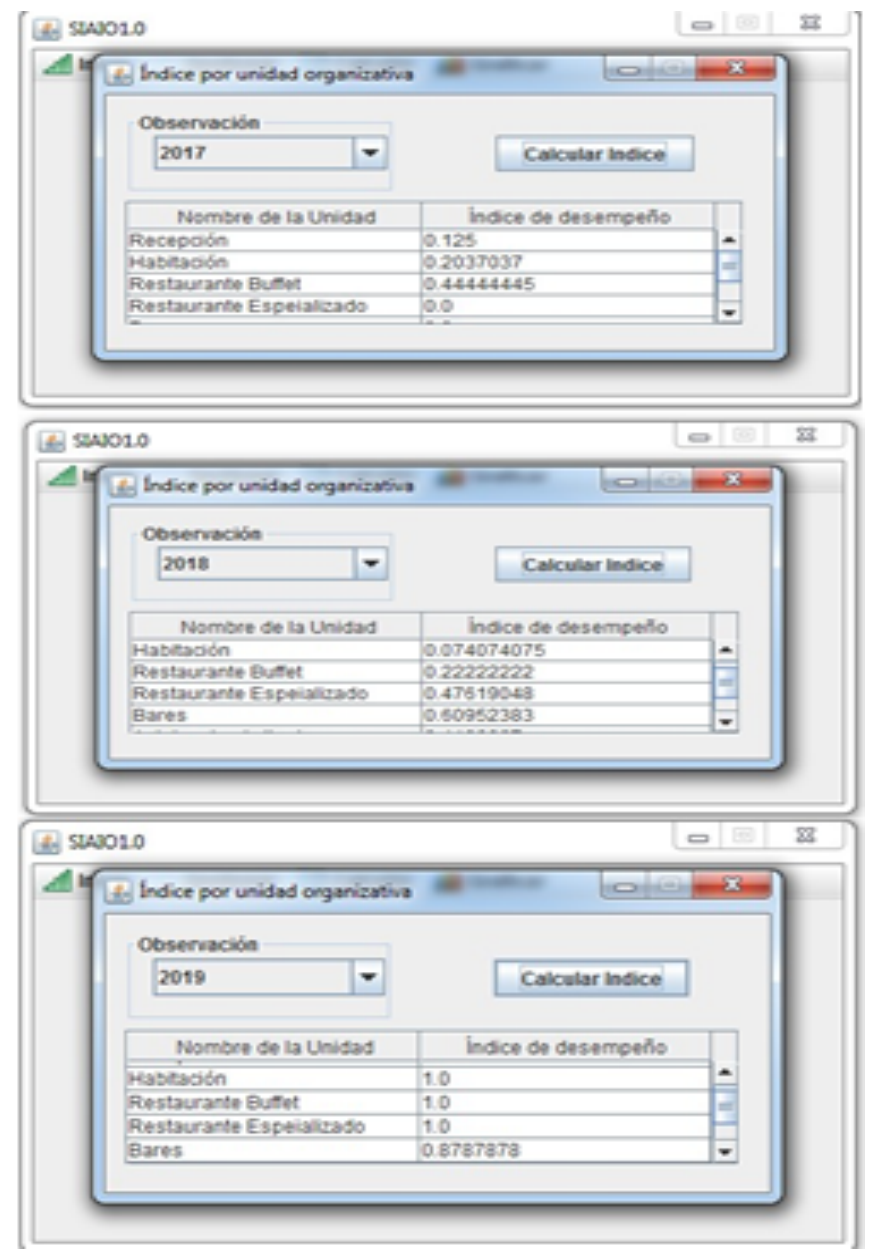

Fuente: Elaboración propia. 
Otra salida que da el software es la visualización del desempeño de las unidades organizativas con su clasificación y como se observa en la figura 6, a medidas que pasan los años el comportamiento del desempeño va mejorando en cada unidad organizativa.

Figura 6: Gráfico del comportamiento del índice por unidad organizativa y su clasificación.
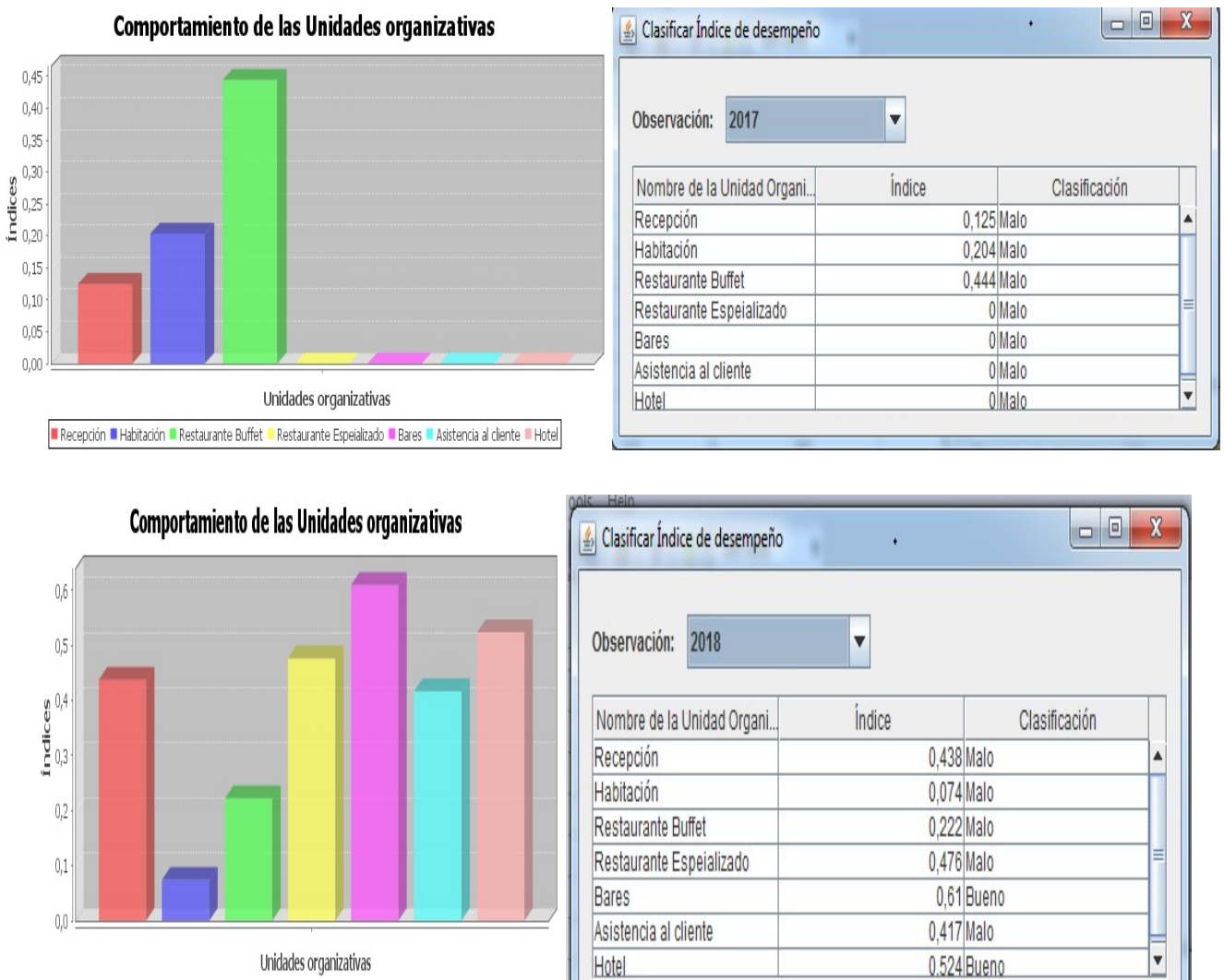

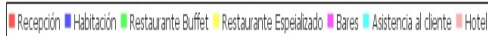
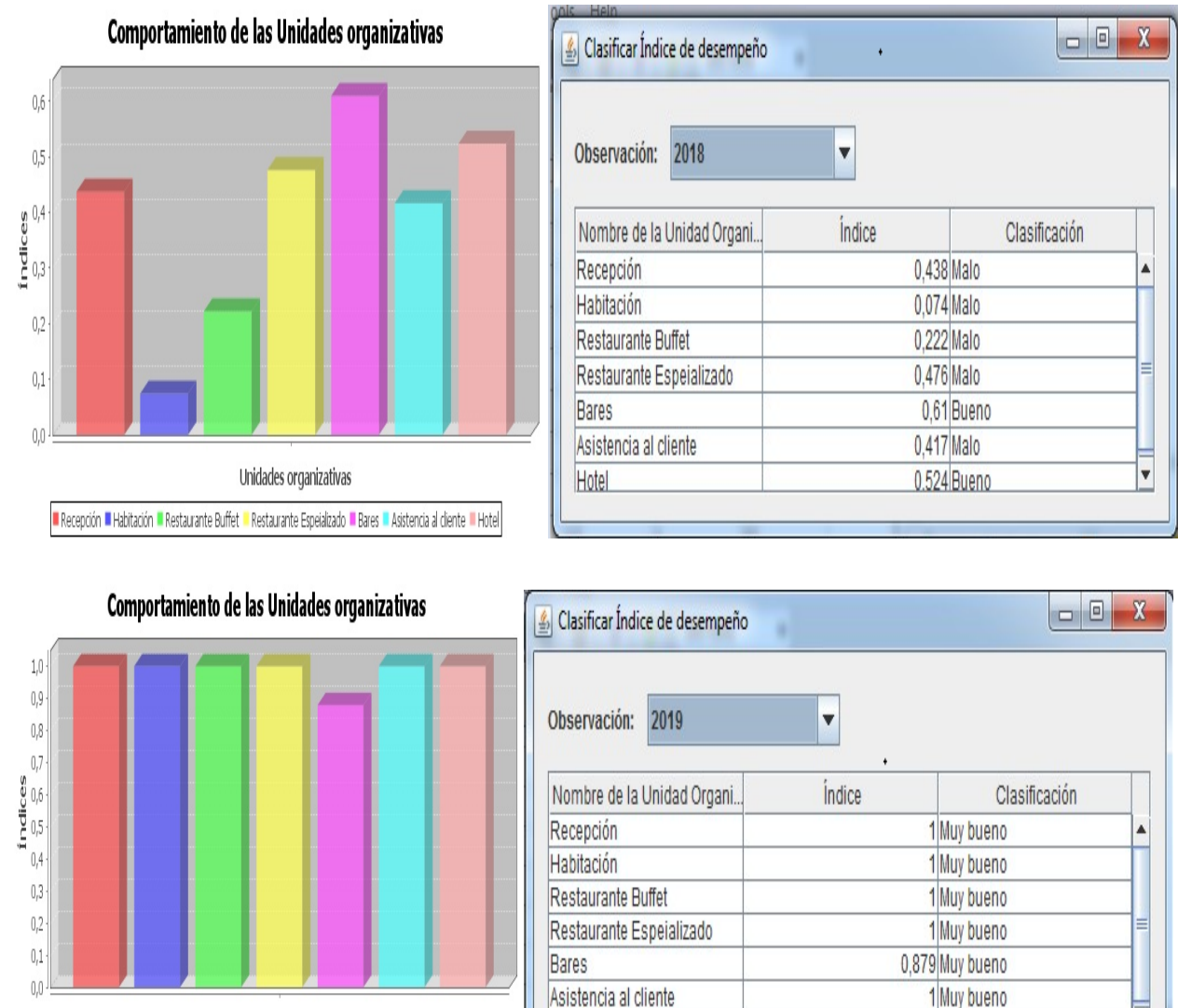

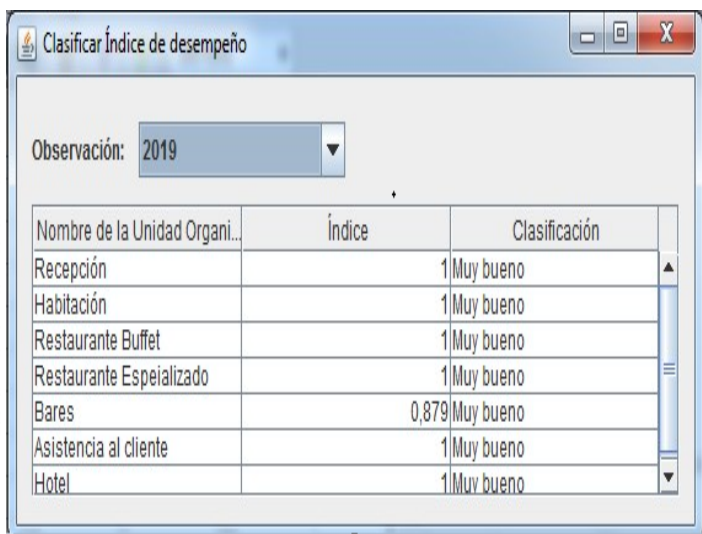

Fuente: Elaboración propia. 
Para hacer un estudio más inteligente sobre el comportamiento del desempeño de indicadores en el hotel, se programó algoritmos de minería de datos para el descubrimiento de conocimiento como son: NNge para las reglas de clasificación (figura 7), Apriori para la regla de asociación (figura 8) y el J48 para generar Árboles de decisión (figura 9).

Figura 7: Interfaz de la aplicación del algoritmo NNge.

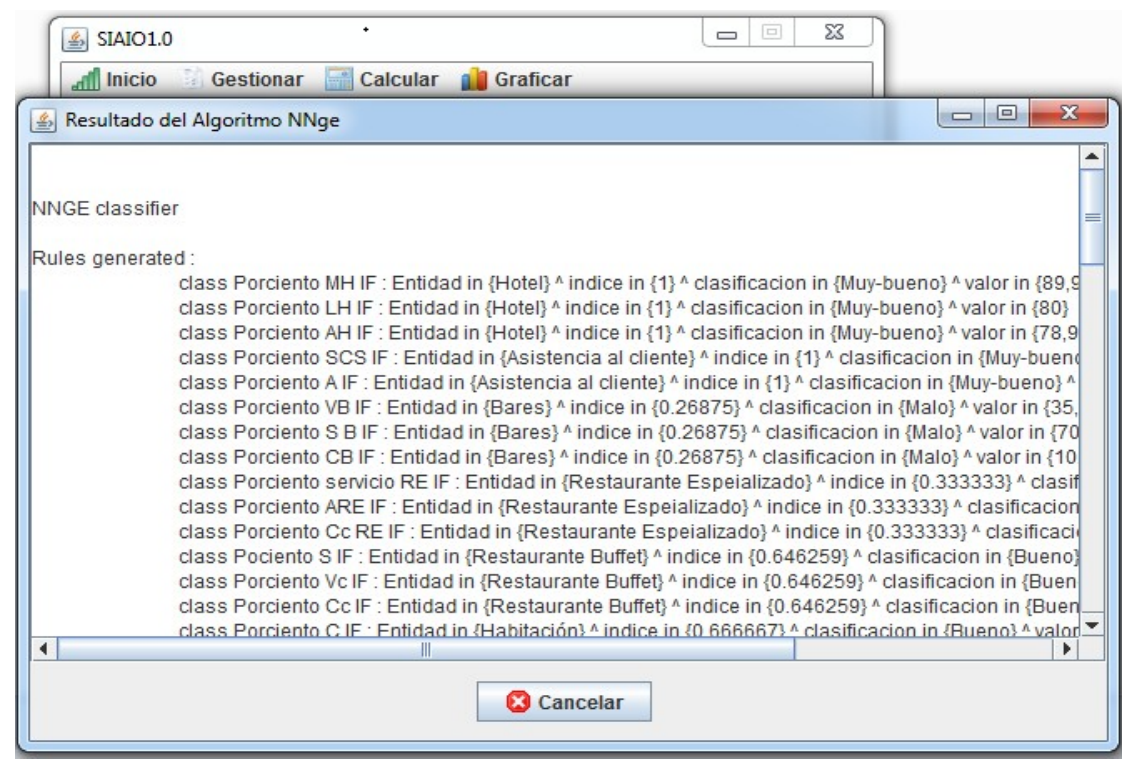

Fuente: Elaboración propia.

Figura 8: Interfaz de la aplicación del algoritmo Apriori.

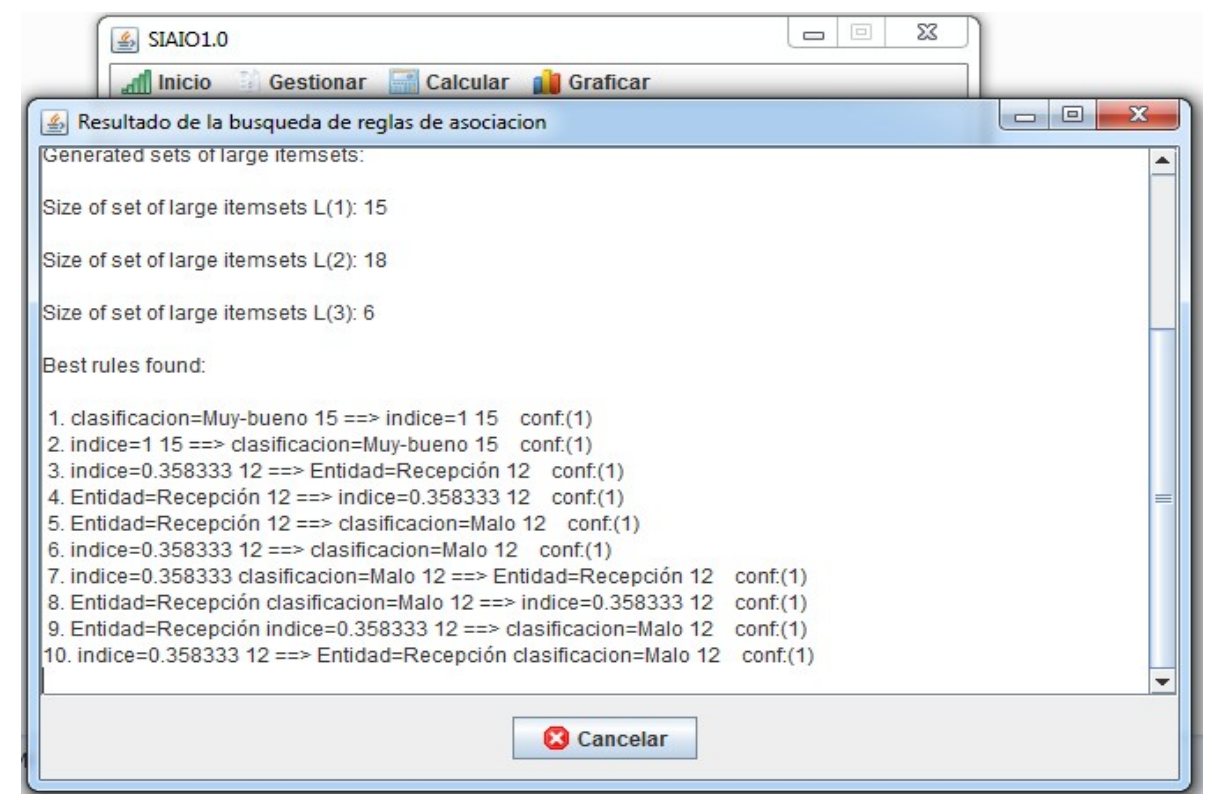

Fuente: Elaboración propia. 
Figura 9: Interfaz de la aplicación del algoritmo J48.

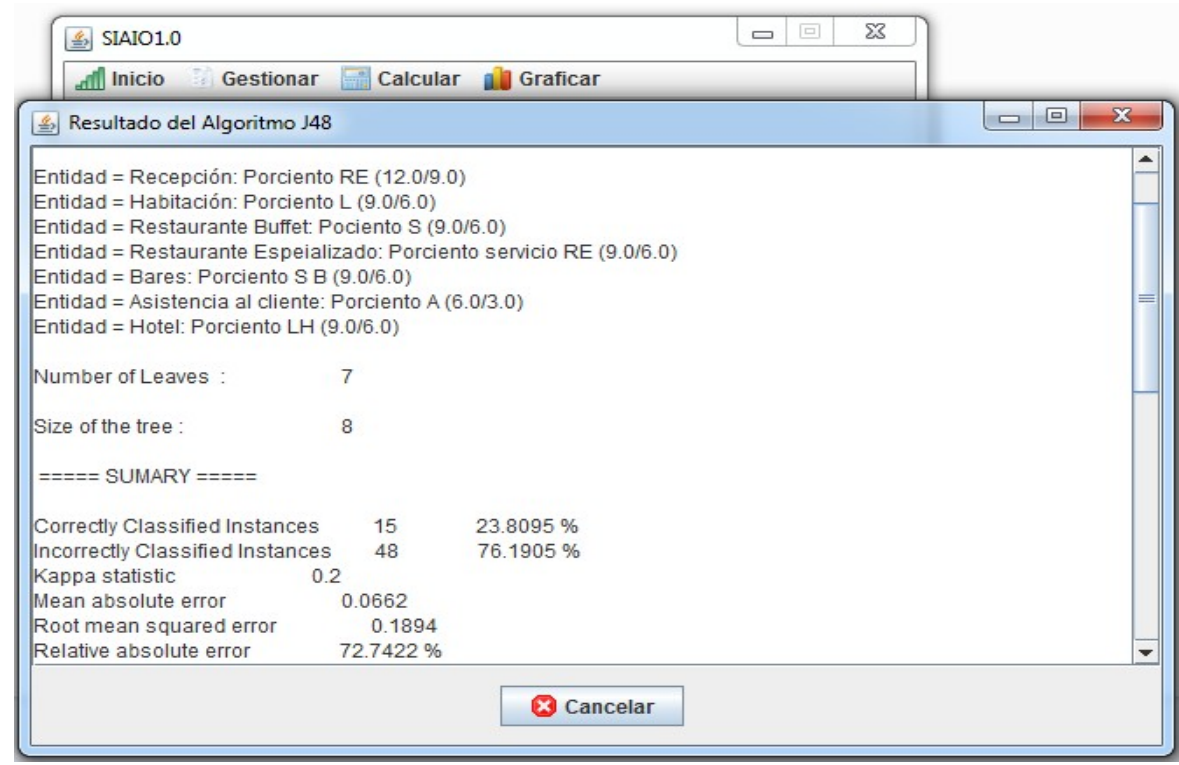

Fuente: Elaboración propia.

\section{Conclusiones.}

En la investigación realizada se arriba a las siguientes conclusiones:

- Se elabora una aplicación informática denominada SIAIO 1.0 que permite el análisis de la información relevante en las unidades organizativas del Hotel Habana Libre, siendo esta una herramienta amigable, sencilla, segura y eficiente.

- El análisis y diseño de la aplicación se llevó a cabo utilizando la metodología RUP, con el lenguaje de modelado UML, representando las características de la aplicación mediante una serie de elementos que conforman esta metodología, esto satisface los requerimientos de consistencia, eficiencia, integridad, flexibilidad y portabilidad de la aplicación.

- La integración de los algoritmos matemáticos y computacionales, con su implementación computacional y los procesos de validación, constituyen una herramienta para el análisis de la información relevante en las unidades organizativas del Hotel Habana Libre.

\section{Referencias bibliográficas.}

Ballester, A., (2012) "Sistema de información para la toma de decisiones comerciales" en GestioPolis. [En línea]. Cuba, disponible en: http://www.gestiopolis.com/sistema-deinformacion-para-la-toma-de-decisiones-comerciales/ 

abril-junio, 2020

Ferrer, M. A. \& Gamboa, T., (2004) Indicadores para el Control de Gestión de Procesos Básicos en Hoteles. Actualidad Contable FACES Año/Vol 7, No. 8, Enero-Julio. Universidad de los Andes, Mérida, Venezuela. Pp. 50-61.

López, C (2004). El Desarrollo Humano y la Equidad en Cuba. Revista Cubana de Salud Pública. ISSN 0864-3466 versión on-line. 2004.

OMT (2016): WTO World tourism barometer: international tourism on track for another strong year. Dpto. de Prensa y Comunicaciones de la OMT. Disponible en: http://www.unwto.org/newsroom/releases/2005/octuber/barometer.html.

Pérez, L (2020). Sistema informático para el análisis de los indicadores turísticos en el Hotel Habana Libre. Tesis en opción al título de Licenciado en Turismo. Facultad de Turismo. Universidad de la Habana. La Habana.91p.

Sandoval, R. A; Mederos, M.; Rosales, D.; De Quesada, E (2007). Contabilidad Nacional. Editorial Félix Varela, ISBN: 978-959-07-0618-9, La Habana, Cuba. 231p.

Sepúlveda, S (2008). Biograma: metodología para estimar el nivel de desarrollo sostenible de territorios. San José. Costa Rica. IICA. 133p.

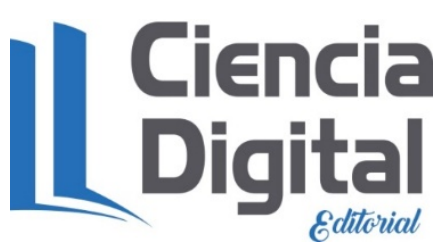


Para citar el artículo indexado

Vázquez Alfonso, Y., Sánchez Arenas, O. J., Ricardo Pérez, L., \& Velasteguí López, L. E. (2020). Sistema informático para el análisis de los indicadores turísticos en el Hotel Habana Libre. AlfaPublicaciones, 2(2), 60-78. https://doi.org/10.33262/ap.v2i2.29

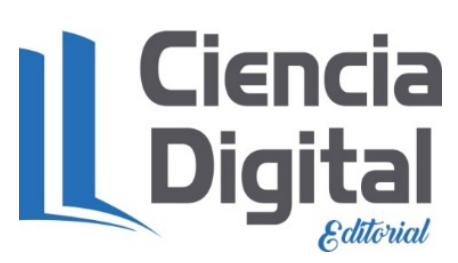

El artículo que se publica es de exclusiva responsabilidad de los autores y no necesariamente reflejan el pensamiento de la Revista Alpha Publicaciones.

El artículo queda en propiedad de la revista y, por tanto, su publicación parcial y/o total en otro medio tiene que ser autorizado por el director de la Revista Alpha Publicaciones.
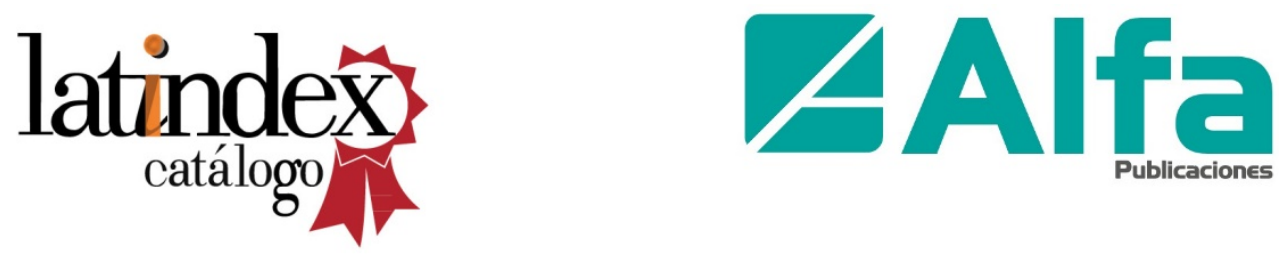